\title{
Early postoperative delirium after hemiarthroplasty in elderly patients aged over 70 years with displaced femoral neck fracture
}

This article was published in the following Dove Press journal:

Clinical Interventions in Aging

6 November 2017

Number of times this article has been viewed

\section{Yi-Hwa Choi' \\ Dae-Hwan Kim² \\ Tae-Young Kim² \\ Tae-Wan Lim' \\ Seok-Woo Kim² \\ Je-Hyun Yoo²}

'Department of Anesthesiology and Pain Medicine, Hallym Sacred Heart Hospital, Hallym University College of Medicine, ${ }^{2}$ Department of Orthopaedic Surgery, Hallym Sacred Heart Hospital, Hallym University College of Medicine, Anyang, Republic of Korea
Correspondence: Je-Hyun Yoo Department of Orthopaedic surgery, Hallym University Sacred Heart Hospital, Hallym University College of Medicine, 22 Gwanpyeong-ro, I70beon-gil, Dongan-gu, Anyang 43I-796, Republic of Korea

$\mathrm{Tel}+82313803770$

Fax +82 31 3803033

Email oships@hallym.ac.kr
Purpose: Postoperative delirium is a risk factor for worse outcome after hip fracture surgery in elderly patients. Postoperative delirium is associated with anesthesia, postoperative pain, and patient factors. We investigated the incidence, predictors, and prognostic implications of postoperative delirium after hemiarthroplasty (HA) in elderly patients with femoral neck fracture. Patients and methods: A total of 356 consecutive patients aged $>70$ years who underwent HA for femoral neck fracture were enrolled. Diagnosis of delirium was made by a psychiatrist based on patient status and an objective scoring system. The patients were divided into 2 categories according to the HA onset time (immediate [ $\leq 24 \mathrm{~h}$ after surgery] vs delayed delirium [ $>24 \mathrm{~h}$ after surgery]) and its incidence, predictors and mortality were evaluated.

Results: Postoperative delirium was diagnosed in 110 patients (30.9\%) during hospitalization. Immediate and delayed delirium occurred in 59 (53.6\%), and 51 (46.4\%) patients, respectively. The independent predictors of immediate delirium included age (odds ratio [OR] 1.47, 95\% CI $0.98-2.23, p=0.066$ ), and general anesthesia (OR 2.25, 95\% CI 1.17-4.43, $p=0.015$ ). The independent predictors of delayed delirium were parkinsonism (OR 5.75, 95\% CI 1.66-19.96, $p=0.006$ ), intensive care unit stay (OR 1.85, 95\% CI 0.97-3.56, $p=0.064$ ), and higher American Society of Anesthesiologists grade (OR 2.33, 95\% CI 0.90-6.07, $p=0.083$ ). On Kaplan-Meier survival analysis, the 2-year survival rate was significantly lower in the immediate delirium group than those in the delayed and control groups $(71.0 \%$ vs $83.6 \%$ vs $87.8 \%$, respectively; $p=0.031)$.

Conclusion: Immediate and delayed delirium after HA for femoral neck fracture had different predictors and immediate delirium was associated with worse prognosis.

Keywords: delirium, elderly patients, femoral neck fracture, hemiarthroplasty

\section{Introduction}

Postoperative delirium is acute brain dysfunction after surgery, which is caused by maladaptation of the brain to the postoperative surgical stress. The incidence and clinical importance of postoperative delirium after hip fracture surgery have increased along with the aging of overall population. ${ }^{1-5}$ Postoperative delirium is associated with high rates of morbidity and mortality in elderly patients undergoing hip fracture surgery. ${ }^{6,7}$ There have been several reports on predictors associated with postoperative delirium, such as advanced age, pre-existing cognitive impairment, and medical comorbidities. ${ }^{8-10}$ It also delays postoperative cognitive and functional recovery, prolongs hospitalization, and increases the burden of medical care and in-hospitalization mortality rate. ${ }^{11,12}$ The early identification of a high-risk group for postoperative delirium in elderly patients is clinically important for preventing associated morbidity and mortality and improving functional outcome. 
Despite its emerging clinical importance, the time course of postoperative delirium and its predictors have not been systematically evaluated after hip fracture surgery in elderly patients. Especially, comparison of its incidence and subsequent results according to the time when delirium occurs after surgery, has not been well evaluated. This difference also has clinical implications, since different approaches would be required in immediate and delayed postoperative delirium, reported as emergence delirium in pediatric patients. In addition, the long-term clinical implication of postoperative delirium has not been well evaluated. Therefore, in the current study, we sought to evaluate the time course, predictors, and long-term clinical significance of postoperative delirium, using data from the prospective cohort of elderly patients undergoing hip fracture surgery.

\section{Materials and methods Study population}

This single-center, observational cohort study was designed to evaluate the "real-world" outcomes of elderly patients undergoing hemiarthroplasty (HA) for femoral neck fracture. The subjects in this study comprised 443 patients who underwent cementless bipolar HA (BHA) for displaced femoral neck fracture between January 2010 and January 2016. Patients $<70$ years of age or with acute pre-operative delirium were excluded from the study. Finally, 356 patients ( 290 women and 66 men) were enrolled in the current study (Table 1). All the data from medical records, patients and their families were gathered retrospectively by independent research personnel using a prospective registry.

Table I Demographic characteristic of the patients

\begin{tabular}{|c|c|c|c|}
\hline Variable & $\begin{array}{l}\text { Immediate } \\
\text { delirium } \\
(\mathrm{N}=59)\end{array}$ & $\begin{array}{l}\text { Delayed } \\
\text { delirium } \\
(\mathbf{N}=5 \mathrm{I})\end{array}$ & $p$-value \\
\hline Age & $81.5 \pm 5.8$ & $81.3 \pm 5.6$ & 0.043 \\
\hline Male & $8(13.6)$ & $8(I 5.7)$ & 0.414 \\
\hline Body mass index & $21.9 \pm 4.1$ & $21.8 \pm 3.6$ & 0.784 \\
\hline Hypertension & $37(62.7)$ & $32(62.7)$ & 1.000 \\
\hline Diabetes & $12(20.3)$ & $13(25.5)$ & 0.627 \\
\hline Previous stroke & $10(16.9)$ & $12(23.5)$ & 0.663 \\
\hline Previous dementia & $12(20.3)$ & $9(17.6)$ & 0.046 \\
\hline Previous parkinsonism & $3(5.1)$ & $6(11.8)$ & 0.005 \\
\hline Depression & $6(10.2)$ & $2(3.9)$ & 0.069 \\
\hline Atrial fibrillation & $6(10.2)$ & $0(0.0)$ & 0.084 \\
\hline Coronary artery disease & $2(3.4)$ & $3(5.9)$ & 0.660 \\
\hline Chronic kidney disease & $2(3.4)$ & $5(9.8)$ & 0.192 \\
\hline Chronic lung disease & $\mathrm{I}(\mathrm{I} .7)$ & $4(7.8)$ & 0.283 \\
\hline Current smoker & $12(20.3)$ & $5(9.8)$ & 0.281 \\
\hline Heavy drinker & II (I8.6) & $7(13.7)$ & 0.694 \\
\hline
\end{tabular}

Notes: Data were presented as mean \pm standard deviation or number (\%). $p$-values in bold indicate statistical significance.
The study protocol was approved by the institutional review board of Hallym University Sacred Heart Hospital (reference numbers: IO13076RG0004993, IRB00005964). All data extracted from patients' records were anonymized and de-identified prior to analysis. Informed consent was waived by the board as it was a retrospective analysis of medical records. Figure 1 shows the CONSORT flow diagram.

All patients underwent a cementless BHA by 2 surgeons (JHY and TYK). Anesthesia was induced and maintained according to the standard institutional regimen. No premedication was administered. Anesthesia was induced with Propofol intravenous, and maintained with sevoflurane under the guidance of bispectral index. Remifentanil $(0.25-0.5 \mu \mathrm{g} / \mathrm{kg}$ per min $)$ infusion was also maintained during anesthesia. According to our institutional protocol, Neostigmine $(0.05 \mathrm{mg} / \mathrm{kg})$ and glycopyrrolate $(0.01 \mathrm{mg} / \mathrm{kg})$ were used to recover from neuromuscular block during all extubation. For patients undergoing spinal anesthesia, 7-12 $\mathrm{mg}$ of $0.5 \%$ hyperbaric bupivacaine was injected into the subarachnoid space. Intravenous sedation using midazolam or dexmedetomidine was used in patients who underwent sedation during spinal anesthesia.

All patients were hospitalized in the orthopedic department for $\sim 2$ weeks after HA. Because the National Public Health system and private health insurance companies cover most of the cost in our country, all patients received standardized medical care postoperatively, and postoperative rehabilitation was initiated 2 days after surgery, and post-acute inpatient rehabilitation was performed consecutively in all patients during the postoperative hospitalization period. These patients were then transferred to the Department of Rehabilitation in our hospital or affiliated rehabilitation centers or nursing facilities for continuous rehabilitation lasting $\sim 2-3$ weeks. Therefore, most of the patients could be followed up to 1 month for postoperative delirium after HA.

\section{Clinical outcomes and adjudications}

The primary outcome of the current study was the occurrence of postoperative delirium. In our institute, we explained the possibility of postoperative delirium to all elderly patients and their guardians before operations and encouraged frequent visits by family members for up to 1 week after surgery to further reduce the risk of postoperative delirium. The patient's cognitive status was routinely evaluated, immediately in the recovery room, and daily in the general ward by the attending surgeon, for 2 weeks after HA. The cognitive status of the patients requiring intensive care unit (ICU) admission was checked at least once a day until discharge. For 


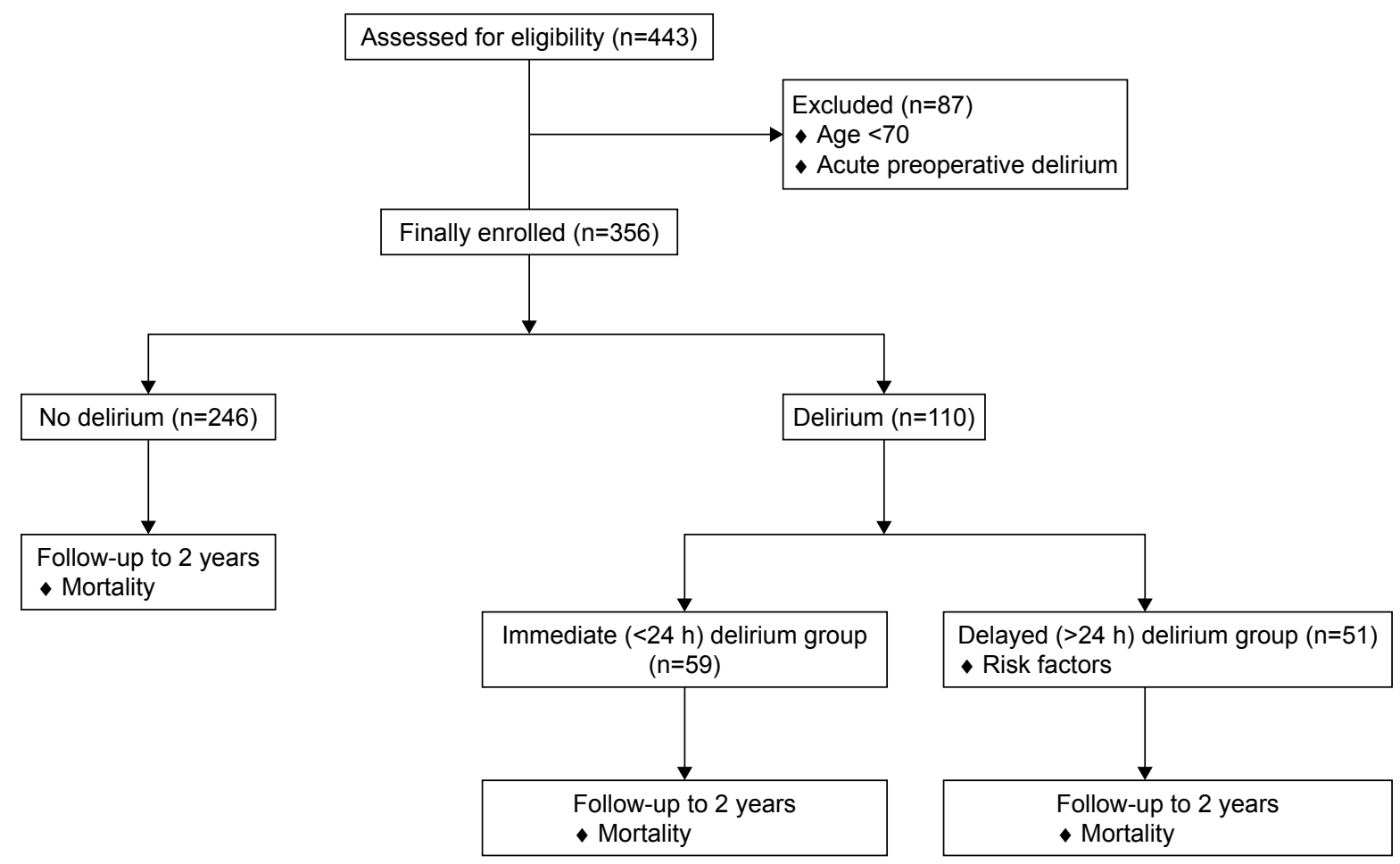

Figure I A CONSORT diagram shows the flow of patients through the study.

patients showing symptoms of delirium such as fluctuation in cognitive function or sense of time, person, or place during the postoperative period, the attending surgeon consulted a psychiatrist for further evaluation and appropriate treatment as per institutional protocol. The study outcome of postoperative delirium was made by an independent psychiatrist using the Confusion Assessment Method (CAM) and CAM-ICU based on the diagnostic criteria of Diagnostic and Statistical Manual-IV. Postoperative delirium was categorized into immediate ( $<24 \mathrm{~h}$ after surgery) or delayed $(>24 \mathrm{~h}$ after surgery) delirium according to the onset time after index surgery. Two-year survival rate was evaluated as the secondary outcome in the current study. The date and cause of death were verified by medical record review and direct or telephone interviews of the patients' families by independent researchers. If the patient was lost to follow-up despite our best efforts, the patient was censored on the last contact.

To evaluate the risk factors of delirium, we analyzed the demographic and perioperative data and laboratory results. As demographic data, we collected the data regarding sex, age, body mass index, underlying comorbidities (hypertension, diabetes, stroke, dementia, parkinsonism, depression, atrial fibrillation, coronary artery disease, chronic kidney disease, chronic lung disease) and current smoking and drinking status. A heavy drinker was defined as $>14$ drinks per week for men, and $>7$ drinks for women. Perioperative data such as American Society of Anesthesiologists (ASA) grade, time from admission to operation, type of anesthesia, operation time, estimated blood loss, transfusion volume, and postoperative complications were collected. Pre-operative laboratory data on hemoglobin, white blood cell count, erythrocyte sedimentation rate, and C-reactive protein levels were also collected. The postoperative factors, such as ICU admission, amount of postoperative drainage, presence of postoperative fever and infection were also collected.

\section{Statistical analysis}

Categorical variables were presented as numbers (\%), while continuous variables were expressed as means and SD. Intergroup comparisons of categorical variables were conducted using the chi-square tests, while those of continuous variables were conducted by Student's $t$ - or the Wilcoxon rank sum tests, as appropriate. Unadjusted event rates were calculated using Kaplan-Meier survival analysis and compared using log-rank tests.

We used Cox proportional-hazards models to examine the association between multiple clinical variables and the risk of delirium. All variables with $p$-value $<0.1$ in the univariate analysis were incorporated in the multivariate model. The independent predictors of delirium were evaluated using backward elimination techniques. The proportional hazards assumption was tested by examination of log-log survival 
curves and partial Schoenfeld residuals and no significant violations were found. All statistical analyses were performed using SPSS ${ }^{\circledR}$ Version 17.0 (SPSS Inc., Chicago, IL, USA) and $p$-values $<0.05$ were considered statistically significant.

\section{Results}

\section{Baseline characteristics}

Postoperative delirium developed in 110 patients (30.9\%) after HA. Among these patients, about half developed delirium within $24 \mathrm{~h}$ after surgery (immediate delirium, 53.6\%) and another half after $24 \mathrm{~h}$ (delayed delirium, 46.4\%). Figure 2 shows the time trend of the rate of delirium after index surgery. The incidence of delirium was highest in the early postoperative period $(<24 \mathrm{~h}, \mathrm{n}=59,53.6 \%)$ and decreased subsequently.

The demographic patient characteristics are summarized in Tables 1 and 2. In the demographic data, the mean age in the delirium group was significantly higher than that of the control group (81.4 \pm 5.7 vs $79.5 \pm 6.7$ years, $p=0.012)$. More patients in the delirium group had underlying dementia ( $p=0.023)$ and parkinsonism $(p=0.014)$ before the injury. Among the perioperative characteristics summarized in Table 2, the ASA grade was higher $(p=0.047)$ and general anesthesia was more frequently used $(p=0.011)$ in the delirium group compared to in the control group.

When postoperative delirium was divided into the 2 groups according to the onset time after index surgery, several baseline and perioperative characteristics differed between the groups. Both the immediate delirium and delayed groups were older ( $p=0.043)$ than the control group. In those with immediate delirium, general anesthesia was performed more

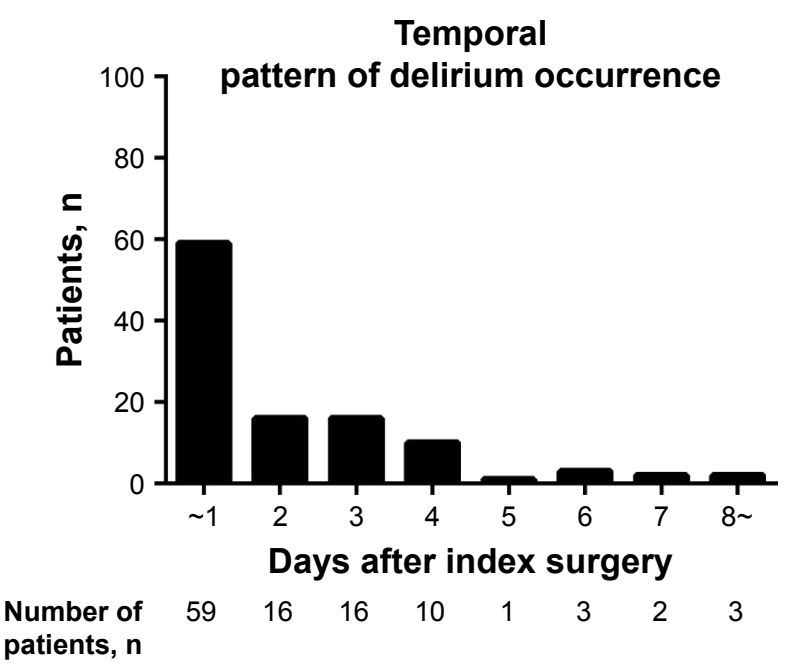

Figure 2 Temporal trend of delirium occurrence after hemiarthroplasty for femur neck fracture.
Table 2 Operative characteristics of the patients

\begin{tabular}{|c|c|c|c|}
\hline Variable & $\begin{array}{l}\text { Immediate } \\
\text { delirium } \\
(\mathbf{N}=\mathbf{5 9})\end{array}$ & $\begin{array}{l}\text { Delayed } \\
\text { delirium } \\
(\mathbf{N}=5 \mathrm{I})\end{array}$ & $p$-value \\
\hline ASA grade & & & 0.118 \\
\hline 2 & 7 (II.9) & $3(5.9)$ & \\
\hline 3 & $51(86.4)$ & $46(90.2)$ & \\
\hline 4 & $\mathrm{I}(\mathrm{I} .7)$ & $2(3.9)$ & \\
\hline Anesthesia & & & 0.024 \\
\hline Spinal & $14(23.7)$ & $15(29.4)$ & \\
\hline General & $45(76.3)$ & $36(70.6)$ & \\
\hline Anesthesia duration & $163.0 \pm 34.5$ & $157.1 \pm 28.7$ & 0.649 \\
\hline Post-operative ICU care & $21(35.6)$ & $26(51.0)$ & 0.032 \\
\hline WBC $(/ \mu \mathrm{L})$ & $8,|| 3.0 \pm 4,070.2$ & $8,226.2 \pm 3,721.1$ & 0.333 \\
\hline $\mathrm{Hb}(\mathrm{g} / \mathrm{dL})$ & $1 \mathrm{I} .7 \pm 1.6$ & $12.0 \pm 1.6$ & 0.319 \\
\hline CRP (mg/L) & $24.3 \pm 35.0$ & $29.5 \pm 40.0$ & 0.775 \\
\hline $\begin{array}{l}\text { Days from injury to } \\
\text { operation }\end{array}$ & $5.7 \pm 7.7$ & $5.7 \pm 5.5$ & 0.174 \\
\hline Operation site & & & 0.412 \\
\hline Neck & 55 (93.2) & 45 (88.2) & \\
\hline Inter-trochanter & $4(6.8)$ & $6(11.8)$ & \\
\hline Operation time (min) & $99.3 \pm 28.0$ & $95.2 \pm 26.8$ & 0.406 \\
\hline Bleeding (cc) & $652.5 \pm 331.3$ & $621.6 \pm 230.1$ & 0.955 \\
\hline Transfusion & $4 I(69.5)$ & $36(70.6)$ & 0.261 \\
\hline \multicolumn{4}{|l|}{$\geq 2$ packed $\mathrm{RBC}$} \\
\hline Pre-operative fever & $50(84.7)$ & $38(74.5)$ & 0.326 \\
\hline Post-operative drainage & $299.2 \pm 252.6$ & $313.7 \pm 206 . \mid$ & 0.318 \\
\hline
\end{tabular}

Notes: Data were presented as mean \pm standard deviation or number (\%). $p$-values in bold indicate statistical significance.

Abbreviations: ASA, American Society of Anesthesiologists; CRP, C-reactive protein; $\mathrm{Hb}$, hemoglobin; ICU, intensive care unit; RBC, red blood cell; WBC, white blood cell.

frequently than in the delayed and control groups ( $p=0.024)$, whereas general anesthesia was performed in similar proportions between the delayed delirium and control groups. Meanwhile, those with delayed delirium had significantly higher ASA grade $(p=0.118)$ and more parkinsonism $(p=0.005)$, which was not evident in the immediate delirium group.

\section{Predictors of postoperative delirium}

The odds ratios (ORs) of each variable were analyzed using a binary logistic regression model (Table 3). In the univariate analysis, age (per 10years; OR 1.44,95\% CI1.04-2.01, $p=0.028$ ), previous dementia (OR 2.18, 95\% CI 1.15-4.12, $p<0.001$ ), parkinsonism (OR 4.28, 95\% CI 1.44-14.21, $p<0.001$ ), ASA grade (OR 2.08, 95\% CI 1.15-3.94, $p=0.001$ ), general anesthesia (OR 1.91, 95\% CI 1.20-3.23, $p<0.001$ ), and ICU care (OR 1.61, 95\% CI 1.01-2.55, $p=0.045$ ) were significant variables. Among these variables, dementia (OR 2.36, 95\% CI $1.07-4.98, p=0.027$ ) and general anesthesia (OR 2.24, 95\% CI $1.19-4.43, p=0.015$ ) were significantly related to immediate delirium, while age (per 10 years; OR 1.47, 95\% CI 0.98-2.24, $p=0.067$ ) showed a positive correlation without statistical 
Table 3 Univariate and multivariate predictors of immediate and delayed delirium after femur neck fracture

\begin{tabular}{|c|c|c|c|c|}
\hline \multirow[t]{2}{*}{ Variables } & \multicolumn{2}{|c|}{ Univariate analysis } & \multicolumn{2}{|c|}{ Multivariate analysis } \\
\hline & OR (95\% Cl) & $p$-value & OR (95\% Cl) & $p$-value \\
\hline \multicolumn{5}{|l|}{ Overall population } \\
\hline Age (per 10 years) & $1.44(1.04-2.01)$ & 0.028 & & \\
\hline Dementia & $2.18(1.15-4.12)$ & $<0.00 \mathrm{I}$ & $2.18(1.13-4.21)$ & 0.021 \\
\hline Parkinsonism & $4.28(|.44-| 4.2 \mid)$ & $<0.00$ I & $3.68(1.18-11.48)$ & 0.025 \\
\hline ASA grade & $2.08(1.15-3.94)$ & 0.001 & $1.93(0.99-3.75)$ & 0.052 \\
\hline General anesthesia & $1.91(1.20-3.23)$ & $<\mathbf{0 . 0 0 I}$ & $1.57(0.93-2.64)$ & 0.089 \\
\hline Days from injury to operation & $0.98(0.94-1.00)$ & 0.074 & $0.97(0.95-1.00)$ & 0.054 \\
\hline ICU care & $1.6 \mathrm{I}(1.0 \mathrm{I}-2.55)$ & 0.045 & & \\
\hline \multicolumn{5}{|l|}{ For immediate delirium } \\
\hline Age (per 10 years) & I.47 (0.98-2.24) & 0.067 & $1.47(0.98-2.23)$ & 0.066 \\
\hline Dementia & $2.36(1.07-4.98)$ & 0.027 & & \\
\hline Parkinsonism & $2.57(0.5 \mathrm{I}-10.79)$ & 0.205 & & \\
\hline ASA grade & $1.56(0.78-3.36)$ & 0.229 & & \\
\hline General anesthesia & $2.24(1.19-4.43)$ & 0.015 & $2.25(1.17-4.33)$ & 0.015 \\
\hline Days from injury to operation & $0.98(0.93-1.00)$ & 0.176 & & \\
\hline ICU care & $1.19(0.65-2.15)$ & 0.567 & & \\
\hline \multicolumn{5}{|l|}{ For delayed delirium } \\
\hline Age (per 10 years) & $1.38(0.90-2.12)$ & 0.139 & & \\
\hline Dementia & $1.98(0.86-4.56)$ & 0.108 & & \\
\hline Parkinsonism & $6.40(1.87-21.87)$ & 0.003 & $5.75(1.66-19.96)$ & 0.006 \\
\hline ASA grade & $2.86(1.20-6.83)$ & 0.018 & $2.33(0.90-6.07)$ & 0.083 \\
\hline General anesthesia & $\mathrm{I} .67(0.87-3.2 \mathrm{I})$ & 0.123 & & \\
\hline Days from injury to operation & $0.98(0.94-1.01)$ & 0.205 & & \\
\hline ICU care & $2.24(1.22-4.13)$ & 0.010 & $1.85(0.97-3.56)$ & 0.064 \\
\hline
\end{tabular}

Note: $p$-values in bold indicate statistical significance.

Abbreviations: ASA, American Society of Anesthesiologists; Cl, confidence interval; ICU, intensive care unit; OR, odds ratio.

significance. For delayed delirium, parkinsonism (OR 6.40, 95\% CI 1.87-21.87, $p=0.003$ ), ASA grade (OR 2.86, 95\% CI $1.20-6.83, p=0.018$ ), and ICU care (OR $2.24,95 \% \mathrm{CI}$ $1.22-4.13, p=0.010$ ) were significantly related to its occurrence. The laboratory findings were not significantly associated with the development of postoperative delirium. The duration of delirium was not significantly different between immediate and delayed delirium (10.3 \pm 7.2 vs $8.6 \pm 7.8$ days, respectively, $p=0.234$ ).

To identify the independent association of variables with postoperative delirium, we established 3 multivariate binary logistic-regression models for overall, immediate and delayed delirium. For overall delirium, the multivariate analysis showed that dementia (OR 2.18, 95\% CI 1.13-4.21, $p=0.021$ ), parkinsonism (OR 3.68, 95\% CI 1.18-11.48, $p=0.025$ ) and ASA grade (OR 1.93, 95\% CI 0.99-3.75, $p=0.052$ ) were independent predictors. General anesthesia (OR 1.57, 95\% CI 0.93-2.64, $p=0.089$ ) was also related to its occurrence, although the relationship was not statistically significant. Further analysis showed different predictors between immediate and late postoperative delirium. General anesthesia (OR 2.25, $95 \%$ CI $1.17-4.33, p=0.015)$ was the only significant predictor of immediate delirium, while age (OR 1.47, 95\% CI
0.98-2.23, $p=0.066$ ) showed clinical implications, although statistical significance was not reached. In terms of delayed delirium, parkinsonism was the only significant predictor (OR 5.75, 95\% CI 1.16-19.96, $p=0.006$ ), while higher ASA grade (OR 2.33, 95\% CI 0.90-6.07, $p=0.083$ ) and ICU care (OR 1.85, 95\% CI 0.97-3.56, $p=0.064$ ) were associated, without statistical significance.

\section{Clinical implication of postoperative delirium on long-term outcomes}

The overall survival rate of the study population in the Kaplan-Meyer analysis is shown in Figure 3A. Overall, patients with delirium showed significantly lower survival rates compared with those without delirium at the 2-year follow-up (77.1\% vs $87.8 \% ; p<0.001)$. In further stratified analysis, the immediate delirium group showed significantly lower survival rates compared with that in the control group ( $71.0 \%$ vs $83.6 \%$ vs $87.8 \%$ for immediate, delayed, and control group, $p=0.031$ for overall comparison; $p=0.014$ for immediate delirium vs control). However, the survival rate of delayed delirium group did not differ significantly from that of the control group ( $83.6 \%$ vs $87.8 \%, p=0.579$ for delayed delirium vs control). Also, there was no clinical significance 

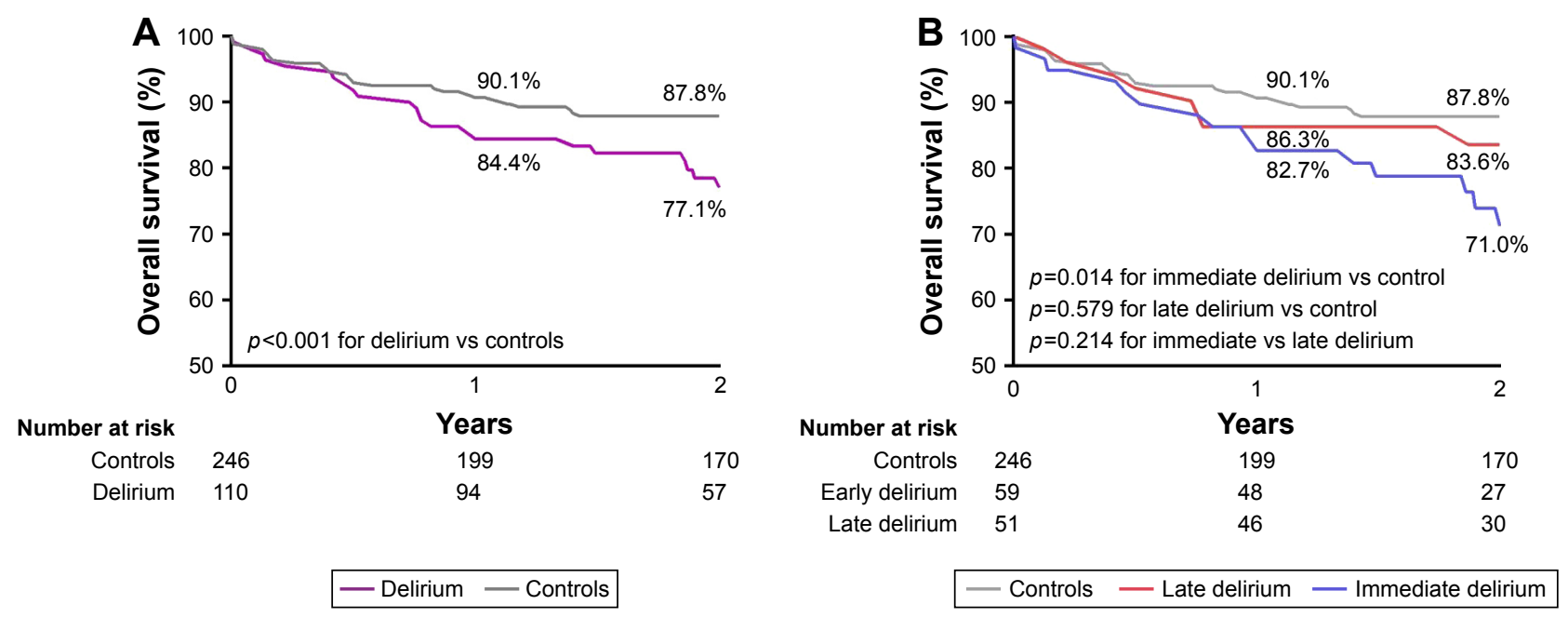

Figure 3 Overall survival rate according to the presence of delirium (A), and stratified analysis of immediate and delayed delirium (B).

in comparison of the immediate and delayed groups $(71.0 \%$ vs $83.6 \%, p=0.214)$.

\section{Discussion}

This retrospective study demonstrated several important findings: 1) the occurrence of delirium was associated with advanced age and several comorbidities; 2) half of the delirium occurred immediately after surgery (within $24 \mathrm{~h}$ ), and another half occurred thereafter; 3) immediate and delayed-onset delirium showed different characteristics and predictors; 4) postoperative delirium was associated with higher mortality during the long-term follow-up, and especially, immediate delirium showed significantly higher mortality than delayed delirium.

This study categorized postoperative delirium into immediate and delayed delirium according to the onset time after surgery. As previous studies used different criteria to define immediate delirium or early postoperative cognitive dysfunction, ${ }^{13-15}$ there has been no unified definition of immediate delirium to date. We divided patients into 2 groups on the basis of postoperative $24 \mathrm{~h}$, because 1 ) postoperative stress and pain would be maximal; 2) residual effect of anesthesia as well as hemodynamic instability would not have fully resolved; and 3) more than half of delirium episodes in the current study occurred during the period within $24 \mathrm{~h}$ after surgery. Our categorization is similar with that of Lee et al, ${ }^{14}$ who reported that delirium occurred most frequently within 2 days after surgery.

Based on our findings, predisposing dementia and parkinsonism, and higher ASA score were significant predictors for patients at increased risk of postoperative delirium. However, immediate and delayed delirium showed different predictors. In patients with immediate delirium, advanced age and general anesthesia were significant predictors. Delirium is not a surprising finding, as it is more prevalent in older individuals because they generally also have neurovascular disease risk factors, higher white matter vascular damage burden, and less cognitive reserve, which may place them at a higher risk for cognitive complications after stressful interventions, including surgery and general anesthesia. Elderly patients have both a reduced ability to respond to stress and to adapt to an abnormal metabolism, a dysfunctional state accompanied by the loss of central cholinergic neurons. ${ }^{16}$ In the presence of acute stressors such as hip fractures and surgical procedures, disturbances to a wide variety of neurotransmitter systems appear to make the elderly more prone to postoperative delirium. ${ }^{17,18}$ Meanwhile, in patients with delayed delirium, underlying parkinsonism and ICU care were predictors. Delayed recovery from index surgical stress would be a plausible cause for the characteristics of delayed delirium. Finally, our study clearly demonstrated the different characteristics, attributable factors, and mortality for immediate and delayed delirium after surgery.

Postoperative delirium delays postoperative functional recovery, prolongs the length of hospital stay, and increases the burden of care and in-hospitalization mortality rate. ${ }^{11,12}$ However, the long-term prognostic importance of postoperative delirium has been rarely reported. Considering this point, our report of higher mortality in immediate delirium may have clinical importance. We believe that the higher mortality rates in the immediate delirium group might have been caused by not only the vulnerability in elderly patients but also a delay of early mobilization and subsequent rehabilitation necessary for satisfactory functional recovery after hip fracture 
surgery. Advanced age of the immediate delirium group compared with the control group may also be a causative factor for higher mortality. The fact that early ambulation and rehabilitation affect the functional outcome associated with morbidity and mortality has been well-known, particularly in elderly patients undergoing hip fracture surgery. ${ }^{19}$ Our result is consistent with a single postoperative registry from Edelstein et al, who reported postoperative delirium within $24 \mathrm{~h}$ after HA influences the mortality rate in elderly patients. ${ }^{20}$ Different prognostic implications of immediate and delayed delirium cannot be fully explained by our limited registry data. However, we believe that advanced age as well as maladaptation to the immediate postoperative surgical stress, reflected in the immediate delirium group, would be markers of vulnerable elderly patients, who are prone to higher long-term morbidity and mortality as well as shortterm. Therefore, a more cautious approach for each type of delirium and evaluation of underlying comorbidities would be demanded for these patients.

The current study has several limitations. First, this is a retrospective analysis. Therefore, it may not assess the direct causal relationship between delirium onset and mortality. Second, as the attending surgeons initially detected symptoms of delirium and consulted a psychiatrist, we may have missed some cases of hypoactive delirium, which could underestimate the number of patients with delirium and affects the categorization between immediate and delayed delirium. Third, this study was conducted to evaluate only the natural course, risk factors of delirium and long-term mortality. We believe that the difference in mortality between immediate and delayed delirium is from the vulnerability of elderly patients and the delayed rehabilitation. However, the role of interventions for prevention or early recovery of delirium should be evaluated in the future.

In conclusion, different characteristics, predictors and prognosis between immediate and delayed delirium were noted in the current study. Immediate delirium in elderly patients $>70$ years of age undergoing HA showed significant different predictors along with higher mortality. Therefore, elderly patients with risk factors of immediate delirium after surgery should be monitored more carefully at each medical visit due to the greater risk of mortality.

\section{Disclosure}

All authors report no conflict of interest in this work.

\section{References}

1. Zywiel MG, Prabhu A, Perruccio AV, Gandhi R. The influence of anesthesia and pain management on cognitive dysfunction after joint arthroplasty: a systematic review. Clin Orthop Relat Res. 2014;472(5): 1453-1466.

2. Zlowodzki M, Tornetta P III, Haidukewych G, et al. Femoral neck fractures: evidence versus beliefs about predictors of outcome. Orthopedics. 2009;32(4):260-267.

3. Foss NB, Kehlet H. Mortality analysis in hip fracture patients: implications for design of future outcome trials. Br J Anaesth. 2005;94(1):24-29.

4. Krenk L, Rasmussen LS. Postoperative delirium and postoperative cognitive dysfunction in the elderly-what are the differences? Minerva Anesthesiol. 2011;77(7):742-749.

5. Ely EW, Shintani A, Truman B, et al. Delirium as a predictor of mortality in mechanically ventilated patients in the intensive care unit. JAMA. 2004;291(14):1753-1762.

6. Edlund A, Lundstrom M, Lundstrom G, et al. Clinical profile of delirium in patients treated for femoral neck fractures. Dement Geriatr Cogn Disord. 1999;10(5):325-329.

7. Marcantonio ER, Flacker JM, Michaels M, Resnick NM. Delirium is independently associated with poor functional recovery after hip fracture. J Am Geriatr Soc. 2000;48(6):618-624.

8. Bryson GL, Wyand A. Evidence-based clinical update: general anesthesia and the risk of delirium and postoperative cognitive dysfunction. Can J Anaesth. 2006;53(7):669-677.

9. Schor JD, Levkoff SE, Lipsitz LA, et al. Risk factors for delirium in hospitalized elderly. JAMA. 1992;267(6):827-831.

10. Raats JW, van Eijsden WA, Crolla RM, et al. Risk factors and outcomes for postoperative delirium after major surgery in elderly patients. PLoS One. 2015;10(8): 0136071.

11. Skrobik Y. Delirium prevention and treatment. Anesthesiol Clin. 2011; 29(4):721-727.

12. Mazzola P, Bellelli G, Brogginni V, et al. Postoperative delirium and pre-fracture disability predict 6 -month mortality among the oldest old hip fracture patients. Aging Clin Exp Res. 2015;27(1):53-60.

13. Ruggiero C, Bonamassa L, Pelini L, et al. Early post-surgical cognitive dysfunction is a risk factor for mortality among hip fracture hospitalized older persons. Osteoporos Int. 2017;28(2):667-675.

14. Lee HJ, Hwang DS, Wang SK, Chee IS, Baeq S, Kim JL. Early assessment of delirium in elderly patients after hip surgery. Psychiatry Investiq. 2011;8(4):340-347.

15. Steinmetz J, Christensen KB, Lund T, Lohse N, Rasmussen LS; for ISPOCD Group. Long-term consequences of postoperative cognitive dysfunction. Anesthesiology. 2009;110(3):548-555.

16. Robertson BD, Rovertson TJ. Postoperative delirium after hip fracture J Bone Joint Surg Am. 2006;88(9):2060-2068.

17. Siverstein JH, Timberger M, Reich DL, et al. Central nervous system dysfunction after noncardiac surgery and anesthesia in the elderly. Anesthesiology. 2007;106(3):622-628.

18. Tucker AM, Stem Y. Cognitive reserve in aging. Structure. 2010;1: 354-360.

19. Gruber-Baldini AL, Zimmerman S, Morrison RS, et al. Cognitive impairment in hip fracture patients: timing of detection and longitudinal follow-up. J Am Geriatr Soc. 2003;51(9):1227-1236.

20. Edelstein DM, Aharonoff GB, Karp A, et al. Effect of postoperative elirium on outcome after hip fracture. Clin Orthop Relat Res. 2004;May(422):195-200. 


\section{Publish your work in this journal}

Clinical Interventions in Aging is an international, peer-reviewed journal focusing on evidence-based reports on the value or lack thereof of treatments intended to prevent or delay the onset of maladaptive correlates of aging in human beings. This journal is indexed on PubMed Central, MedLine,

CAS, Scopus and the Elsevier Bibliographic databases. The manuscript management system is completely online and includes a very quick and fair peer-review system, which is all easy to use. Visit http://www.dovepress. com/testimonials.php to read real quotes from published authors. 